\title{
An optimisation framework for designs for additive manufacturing combining design, manufacturing and post-processing
}

\author{
Anton Wiberg, Fohan Persson and Fohan Ölvander \\ Linköping University, Linköping Sweden
}

\begin{abstract}
Purpose - The purpose of this paper is to present a Design for Additive Manufacturing (DfAM) methodology that connects several methods, from geometrical design to post-process selection, into a common optimisation framework.

Design/methodology/approach - A design methodology is formulated and tested in a case study. The outcome of the case study is analysed by comparing the obtained results with alternative designs achieved by using other design methods. The design process in the case study and the potential of the method to be used in different settings are also discussed. Finally, the work is concluded by stating the main contribution of the paper and highlighting where further research is needed.

Findings - The proposed method is implemented in a novel framework which is applied to a physical component in the case study. The component is a structural aircraft part that was designed to minimise weight while respecting several static and fatigue structural load cases. An addition goal is to minimise the manufacturing cost. Designs optimised for manufacturing by two different AM machines (EOS M400 and Arcam Q20+), with and without post-processing (centrifugal finishing) are considered. The designs achieved in this study show a significant reduction in both weight and cost compared to one AM manufactured geometry designed using more conventional methods and one design milled in aluminium.

Originality/value - The method in this paper allows for the holistic design and optimisation of components while considering manufacturability, cost and component functionality. Within the same framework, designs optimised for different setups of AM machines and post-processing can be automatically evaluated without any additional manual work.
\end{abstract}

Keywords Additive Manufacturing, Design for Additive Manufacturing, Optimisation, Multidisciplinary Design Optimisation, Computer aided design

Paper type Research paper

\section{Introduction}

Design for Additive Manufacturing (DfAM) is a category of methods and knowledge that aids the design process of components by fully using the advantages of AM. AM has several advantages over traditional manufacturing, including the manufacturing of small quantities/individual customisation, part consolidation, the creation of lightweight designs and the possibility to create more efficient designs (Gao, 2015; Gibson et al., 2015; Klahn et al., 2015). Traditional design methods, tools and software are often not capable of supporting a design process that fully exploits the possibilities of AM (Simpson, 2017). The difficulty of using the potential, and understanding the limits, of AM complicates the introduction of AM into industry.

Different methods that use optimisation to overcome the problem are proposed in research. Examples of focus areas for that kind of research are Topology Optimisation (TO) of the geometry (Liu and Ma, 2016; Ranjan et al., 2017), lattice optimisation (Gorguluarslan et al., 2017), optimisation of build direction and placement (Das et al., 2015), slice and manufacturing path

The current issue and full text archive of this journal is available on Emerald Insight at: https://www.emerald.com/insight/1355-2546.htm

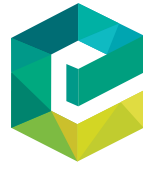

Rapid Prototyping Journal

27/11 (2021) 90-105

Emerald Publishing Limited [ISSN 1355-2546]

[DOI 10.1108/RPJ-02-2021-0041] optimisation (Jin et al., 2017), process optimisation (Liu and Rosen, 2010), material/material properties refinement (Guessasma et al., 2015) and the development of better post-processing processes (Kahlin, 2020). Most of the existing research is limited to one or a few of these categories and therefore does not involve all the important perspectives in a design process.

This paper aims to present a holistic method that bridges disciplines from geometry optimisation to post-processing selection into the same optimisation framework. The presented design framework automatically uses knowledge of AM and postprocessing to prepare a geometry for manufacturing e.g. by addition of stock material that compensates for manufacturing and post-processing. Computer-aided engineering (CAE) models uses

\footnotetext{
(C) Anton Wiberg, Johan Persson and Johan Ölvander. Published by Emerald Publishing Limited. This article is published under the Creative Commons Attribution (CC BY 4.0) licence. Anyone may reproduce, distribute, translate and create derivative works of this article (for both commercial and non-commercial purposes), subject to full attribution to the original publication and authors. The full terms of this licence may be seen at http://creativecommons.org/licences/by/4.0/legalcode
}

This work is performed as a part of the project AddMan. AddMan is a part of Clean Sky 2 Joint Undertaking under European Union's Horizon 2020 research and innovation programme under grant agreement No. 738002.

Received 24 February 2021

Revised 9 August 2021

Accepted 11 September 2021 
the created geometries to evaluate manufacturability and manufacturing cost in parallel to structural performance. The method enables comparison of different AM and post-processing setups, in addition to geometry variations enabled by a parametric CAD model. Contribution to the research field of DfAM is a synthesised and holistic method supporting the involvement of $\mathrm{AM}$ in the design process. While the individual design disciplines are based on existing technologies, this work also contributes with how they need to be adopted to fit within a holistic design automation framework.

The method is applied to a case study where a structural aircraft component is designed and manufactured. In the case study, the framework for automatic design evaluation is implemented into a multi-disciplinary optimisation (MDO) (Simpson and Martins, 2011) framework, aiming to optimise both the performance of the final product and its manufacturing. The outcome of the optimisation is not just one optimal design but several designs that are optimal (in term of performance and manufacturing cost) for different setups of AM machines and post-processing methods. In addition to this optimisation, the knowledge reuse in the method enables it to automate the export of geometrical models with added material that compensates for manufacturing and post-processing.

The work presented in this paper follows a methodology similar to Design Research Methodology (DRM) (Blessing and Chakrabarti, 2009), to formulate, evaluate and analyse a design method. The design method is formulated based on data collection in the form of literature reviews and previous experience from research projects carried out in collaboration with industry. A condensed review of DfAM research is presented in Section 2. The formulated design method is presented in section 3. The design method has been implemented within a computer framework that utilises the proposed method to support the design process.

A case study is used to evaluate the method and framework. This case study consists of a component from an aircraft cargo door structure. This component was selected because the aeronautical industry was one of the early adopters of AM, and this is a typical aircraft component. It is designed to minimise the weight and manufacturing cost while structural loads and manufacturing rules are considered. This is presented in Section 4, together with a comparison between the designs achieved in this study and existing design alternatives. In Section 5, the design method is analysed and discussed based on experiences from the case study. An overview of the four steps in the methodology is presented in Figure 1.

\section{Design for additive manufacturing}

DfAM includes a variety of research, methods, software and information which aid the design process from divergent angles (Gao, 2015). Several different methods and categorisations of DfAM exist (Wiberg et al., 2019a; Yang and Zhao, 2015; Kumke et al., 2016; Laverne et al., 2014; Rosen, 2014), which contain a collection of different methods, information and other types of design support. From a design engineer's perspective, DfAM can be split into four categories (component selection and ideation, design and optimisation, AM preparation and postprocess preparation), as shown in Figure 2. The first category focuses on component selection and assembly design. Research in the second category focuses on design support, where computer support in term of CAD and optimisation methods and tools dominate. The third deals with rules, software and simulation methods that aim to prepare and verify the success of the manufacturing. Within the fourth and final phase, the component is adapted and prepared for postprocessing, including removal from the build plate, removal of support structures, heat treatment and potential surface improvement methods.

\subsection{Component selection and ideation}

The earliest usage of AM was to create prototypes, but today $\mathrm{AM}$ is used for prototypes, final products and tools or moulds (Klahn et al., 2015). The proposed method focuses on end products, for which AM has four advantages compared to traditional manufacturing. The first is integrated design with a focus on reducing the number of parts in a system. This can lead to a reduction in part count with the positive effects of reduced risk of errors and reduction in manufacturing cost. Integrated design can also make it possible to integrate functions that cannot be manufactured using traditional

Figure 1 The four-step methodology applied in the study

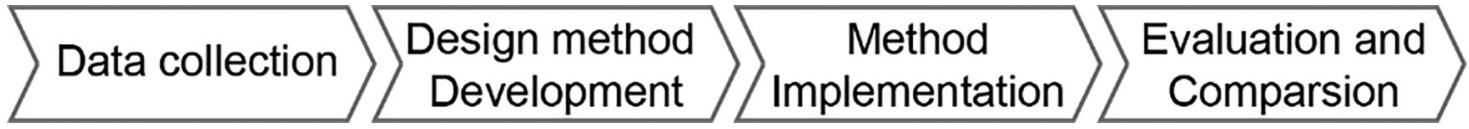

Figure 2 Four steps in the design for additive manufacturing process

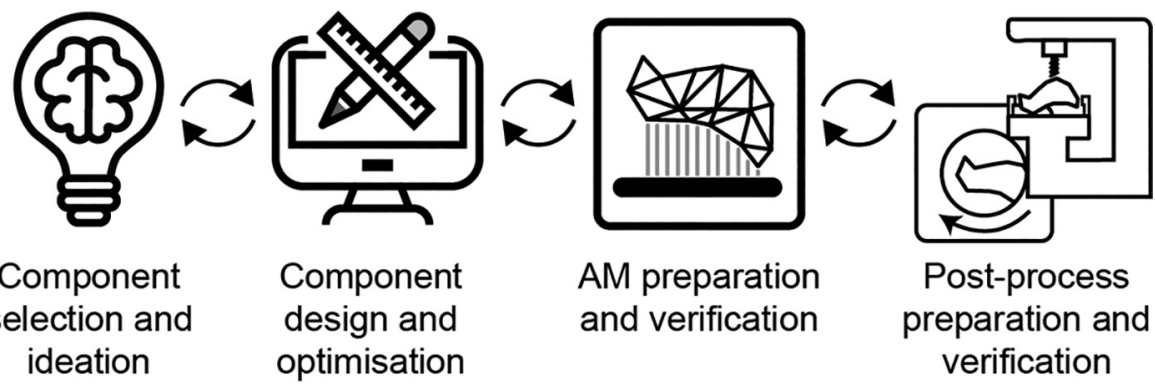


methods. A second advantage of AM is the possibility to create customised components. The third advantage is to create a lightweight design compared to conventional manufacturing. The cost can also be reduced due to using less material. As a fourth advantage, complex structures with optimised channels or other integrated functionalities, can be manufactured (Klahn et al., 2014).

Methods that aid the ideation include design heuristics, which are used as inspiration during early design phases (Blösch-Paidosh and Shea, 2019; Lindwall and Törlind, 2018). Another, similar, method is to increase innovation in design principles (Perez et al., 2019), which in addition to inspiration also include manufacturing variables (Mani et al., 2017).

\subsection{Component design and optimisation}

One of the main trends in optimisation for AM is topology optimisation (TO). TO is an optimisation method in which the geometry is optimised by varying the density of mesh elements in a space. The result takes the form of a mesh density map (Christensen and Klarbring, 2009). The output from TO is, in most cases, a boundary representation of the optimised mesh. TO is attractive because it directly satisfies one of the possibilities of AM (weight optimisation). The method is also simple in that it only needs a meshed design space, boundary and load conditions and defined constraints and objectives. Historically, TO has not been very useful for the design of end components due to the challenge of manufacturing the optimised result (Zegard and Paulino, 2016). The freeform possibilities of AM have revolutionised the usage of TO now that it is possible to manufacture complex shapes. Some challenges to fully enable the usage of TO for the direct creation of parts still exists, including consideration of overhang angle and other design rules, the addition of other constraints or objectives than structural characteristics and manipulation of the geometry due to external or late introduced requirements (Zegard and Paulino, 2016). TO filters for design rules are constantly developing, including overhang minimisation, anisotropic material behaviour and others (Liu, 2018).

In contrast to $\mathrm{TO}$, where the final geometry is difficult to change, parametric design can be used to create geometries which are easy to change or adapt to new contexts, and where it is possible to integrate knowledge (Amadori et al., 2012). TO results can be interpreted into a parametric model by building a skeleton with the TO result as base and then adding a skin around it (Asadollahi-Yazdi et al., 2017). To create a model that integrates and reuses knowledge while different variations of the geometry are achieved, a master model (MM) approach, which uses CAD templates and object oriented programming, can be used (Biedermann and Meboldt, 2020; Wiberg et al., 2019b).

MDO is a field of research that applies different numerical optimisation techniques to design engineering systems and components involving multiple disciplines (Simpson and Martins, 2011). MDO enables a systematic design approach in which optimisation and exploration are used to solve design problems where multiple variables and disciplines affect each other (Vandenbrande et al., 2006). In contrast to, multidisciplinary and multi-objective optimisation can be used to combine several aspects of the design process. Examples are: minimised support structure and manufacturing error (Das et al., 2015), build time and manufacturing error (Das, 2016). Another example of multi-objective AM optimisation is Salonatis and Zarban. (2015), who use multi-criteria decisionmaking to evaluate several objectives in AM design. In addition to only changing the AM setup, Asadollahi-Yazdi et al. (2018) add geometrical changes by morphological geometry variations combined with manufacturing parameters to minimise manufacturing time and material use while considering structural and surface roughness constraints. Wiberg et al. (2018) vary a parametric CAD model and build direction while minimising component weight and support structure.

\subsection{AM verification}

Verification that it is possible to manufacture the design using $\mathrm{AM}$ can be performed via either geometrical design rules (Adam and Zimmer, 2015) or computational simulation approaches (Bikas et al., 2016). The geometrical design rules include recommendations for e.g. overhang angle where a support structure is needed, minimum thicknesses or gaps and how material translation can be designed without failing (Adam and Zimmer, 2015; Thomas, 2009; Kranz et al., 2015). By formalising the rules as functions of factors such as material and AM machine, they can be implemented into a framework which automatically evaluates them (Mani et al., 2017; Rudolph and Emmelmann, 2017).

Computational simulation approaches are in general more accurate and can be used to predict deformations and failures of prints (Bikas et al., 2016). On the downside, the simulation is often computationally expensive and requires expensive software licenses.

\subsection{Post-process preparation and verification}

Most AM processes and components require post-processing after the AM build, the most obvious being the removal of surrounding powder, removing the component from the build plate and support material removal. Other reasons for the postprocessing of $\mathrm{AM}$ components are to ensure tolerances of geometrical features (Schnabel et al., 2017) and improve material properties (Kahlin, 2020).

The tensile properties of materials, in terms of yield strength (YS) and ultimate tensile strength (UTS), are relatively similar to those of components manufactured using conventional methods such as casting (Lewandowski and Seifi, 2016). However, the fatigue properties of AM manufactured components are generally worse than those of corresponding conventional material due to rough surfaces and internal defects (Kahlin et al., 2017). Materials manufactured by laser and electron-beam also show significant differences, with electron-beam material having greater surface roughness and therefore also worse fatigue life. Different post-processing methods, such as laser-polishing shoot peening, linishing and centrifugal finishing, as well as heat treatment, have shown potential to increase the fatigue life (Kahlin, 2020). As for AM, the different post-processing methods come with design rules that need to be respected.

\section{Proposed method}

The proposed design method bridges the three design steps: Component design and optimisation, AM preparation and 
verification, and Post-process preparation and verification presented in Section 2. Hence utilising a holistic design process in which geometry, manufacturing and post-processing are optimised in the same framework. Central to the proposed design process is a $M M$, which contains geometrical information, as well as component knowledge. Component knowledge is used to enable the export of geometrical information adapted for evaluating different design aspects. As the geometry of the MM is parametric, it is easy to manipulate in the optimization framework. A database with explicit knowledge, including material data and design rules, is used to support the manufacturing and CAE evaluation. Based on AM, post-process and material selection, the database returns the specific design rules and material properties applicable to the design. These design rules are used in the manufacturing evaluation, during which the cost and feasibility of the design is evaluated. The evaluated geometry is compensated for manufacturing and post-processing based on the component knowledge and the manufacturing setup. Final performance of the design is evaluated using $C A E$ analysis where geometrical information from the $M M$ and material properties enable the evaluation.
The design method enables evaluation of divergent designs manufactured by different $\mathrm{AM}$ and post-processes. As an extension, an optimisation engine can be used to automatically create, evaluate and optimise designs within an MDO framework. The optimisation engine controls the process where the design variables are varied and the responses from the CAE models are considered. This section describes the individual design disciplines (MM, AM database, manufacturing evaluation and CAE analysis), in Section 4, the framework is implemented in a case study and an MDO software is used for optimisation. An overview of the design method is presented in Figure 3.

The proposed method described in Figure 3 is software independent; however, in this paper it is implemented in a computer system that connects different existing software, a specifically developed database, with code for manufacturing evaluation and export functions. The system can potentially be expanded to integrate several alternative software packages, but so far it is limited to CATIA V5 as both CAD software and finite element solver, the database is implemented in SQLite and manufacturing evaluation is based on geometrical design rules implemented in the manufacturing model. Furthermore,

Figure 3 Information flow of the proposed method

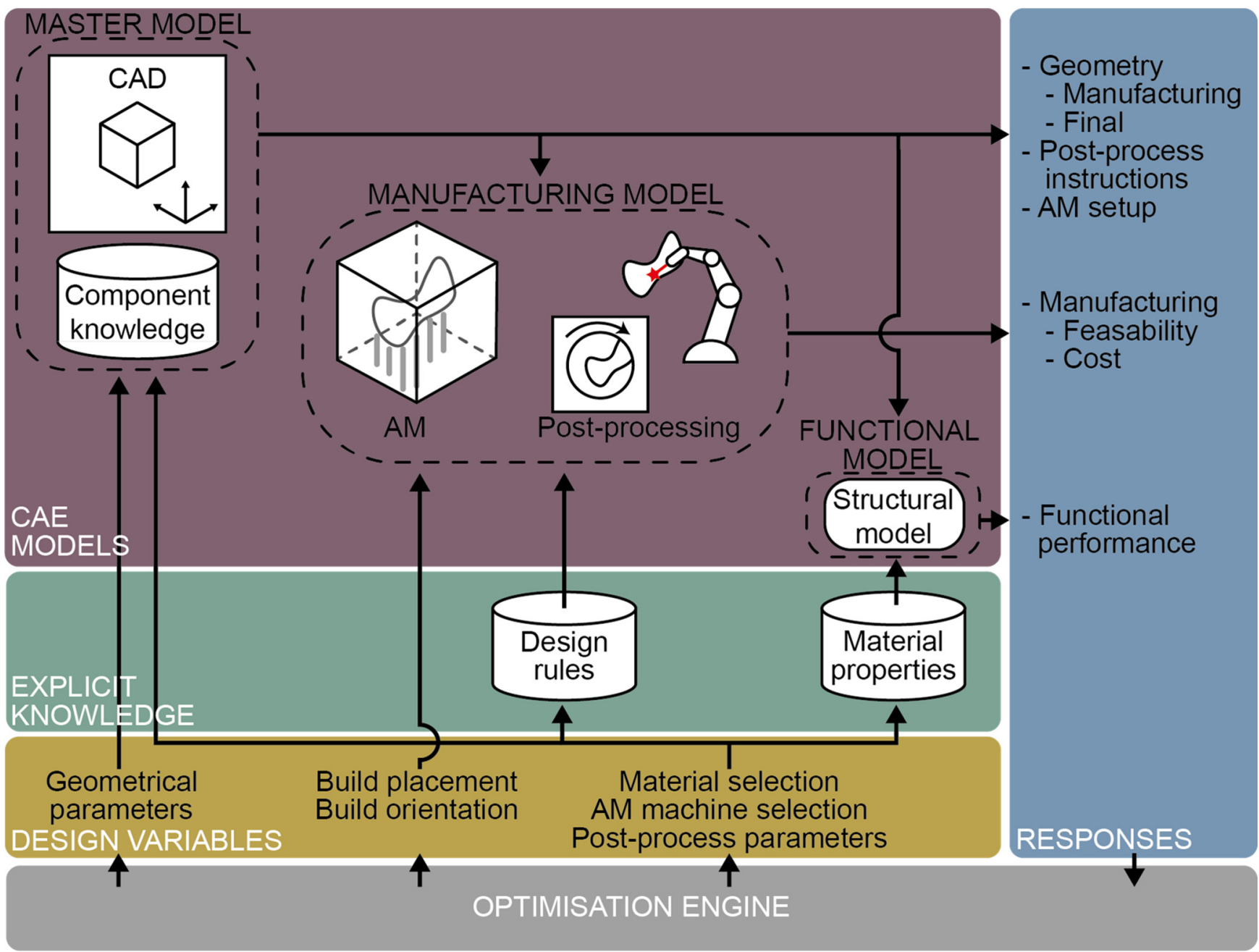


in the case study the optimization engine mode FRONTIER is used to orchestrate the optimisation.

\subsection{Master model}

The aim with the MM is to create a model which comprises the knowledge necessary to automate downstream computer analyses in the DfAM process. By embracing geometrical, functional and manufacturing information in the model, geometrical and other information necessary to perform CAE evaluations and prepare manufacturing are exported. A CAD system is used for creation and saving a parametric CAD model which enables variations based on geometry design variables. High-level CAD templates (Amadori et al., 2012) which include geometrical elements such as planes, points, sketches and surfaces as well as logical rules and design parameters are used to build the CAD model. Selection of which CAD template that is added control the topology in the geometry while the parameters within the templates control morphological changes of the geometrical elements within the template. A template can be added to the component by referencing to one or several points or planes in the CAD model.

Functional decomposition was used to break down the intendent case study component into subfunctions, solutions of the subfunctions were used as base during the development of CAD templates. Example of solutions are different kinds of attachment types and structural sections used to connect the attachments. In the MM, each solution is represented by an object-oriented programming class where attributes contain information of parameter boundaries and other types of additional information regarding the solution. An example is the required tolerances (and thereby also post-processing operations) of a specific feature in the component. When geometrical features are added, this information is added to a database in parallel with the CAD information. Each class contains methods for instantiation of CAD templates linked to the solution. In addition, each class also contains export functions which utilises the knowledge captured in the database in order to create a variation of the geometry adapted to different CAE evaluations (Wiberg et al., 2019b). Implemented in the system is functions which allows export of models adapted for structural verification, manufacturing and how the final geometry will look like, this is presented in Figure 4. Figure 5 show a closer look on how a rivet attachment compensated for manufacturing and post-processing can look like.

For creation of the MM the user needs to manually create reference points in the CAD software and then connect the references to the different functional solutions. Based on this the geometry and parameters are created in the CAD system, and the additional knowledge is saved in the database.

\subsection{AM database}

The MDO framework includes AM databases, which are used to extract information for the specific manufacturing setup chosen. This includes two separate databases, one containing geometrical design rules for the manufacturing and one containing material properties.

\subsubsection{Design rules}

The design rules database is based on commonly accepted geometrical design rules, formalised to enable their saving and reuse within a cohesive structure. Rules for AM and postprocessing machines are integrated into the same database and are automatically evaluated during the manufacturing evaluation. Within the database, parameters for the specific AM machines and post-processing processes are saved and extracted for use during the evaluation of the rule. The rules are defined such that, for one rule, a fixed number of parameters are extracted. A machine selection is used as input to the database where the parameters for that machine are coupled to specific rules. An excerpt from the design rules implemented within the framework is presented in Table 1. Each row represents a design rule, including a name, description of the rule and the parameters depending on the rule. In the machine columns, the output parameters for the different machine choices are presented. The database includes two AM machines and one post-process selection. Where no parameters are stated, the corresponding rule is not applicable for that specific machine.

\subsubsection{Material properties}

The material database is used to save the properties of materials manufactured by AM and thereafter post-processed to improve these properties. Input to the database is selection of an AM machine and whether post-processing is to be used. Output is material properties categorised based on the kind of CAE evaluation in which they are to be used. For them to be useful, the properties are simplified into parameters that can be used directly in commercial CAE software. The eventual influence of manufacturing settings or geometric shape on the material properties are also neglected.

There are two types of material properties, static and fatigue properties. The static properties are fixed parameters in the form of Young's modulus, Poisson's ratio, density, yield and ultimate strength. Fatigue behaviour of a material is instead described in the form of an S-N curve, where the fatigue life of a material for a certain stress level is described. In the database, the maximum stress allowed for a defined number of load cycles is a function based on measured data. The static properties in the database are from the machine manufacturers, while the fatigue properties are calculated from Kahlin et al. (2020). An example the material properties for Ti6Al4V is given in Table 2 .

\subsection{Manufacturing Evaluation}

In the manufacturing evaluation, the feasibility of manufacturing is evaluated based on the design rules from the AM database. In addition, the cost of manufacturing is predicted using a cost model that estimates the manufacturing cost based on AM machine selection, data from the manufacturing evaluation and post-process selection.

Design rules are evaluated with functions where the manufacturing geometry, the design rule parameters from the database and specific build parameters (such as build placement and orientation) are used as input. Based on this input, the function calculates a Manufacturing Feasibility (MF) index (Mani et al., 2017), which represents how well the design is fit for manufacturing. In this framework, a lower 
Figure 4 Overview of information flow from the Master Model

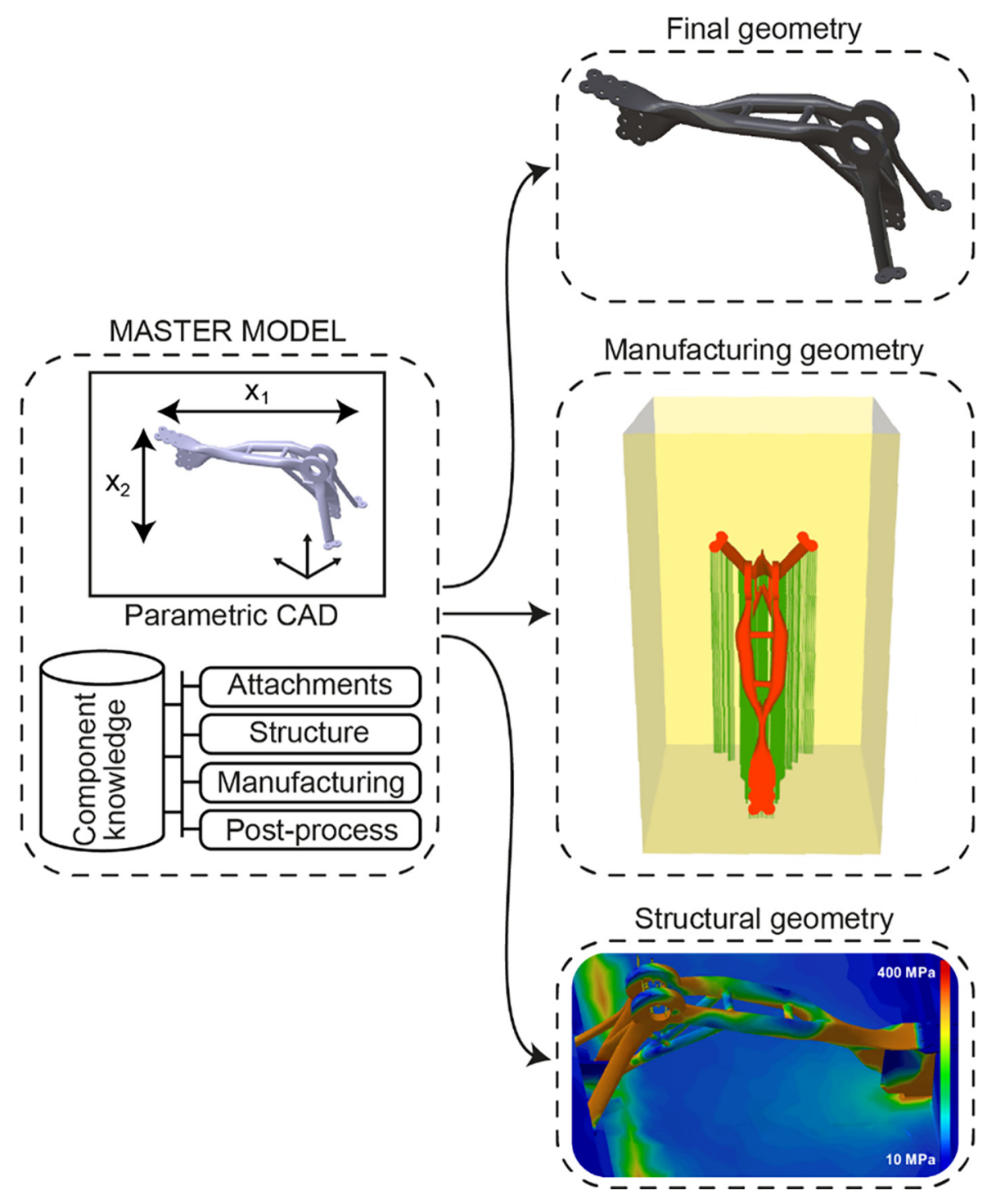

Figure 5 Example of a model exported for manufacturing where the final geometry has been compensated with material that will be removed during postprocessing (e.g. centrifugal finishing) and machining (e.g. drilling and milling)

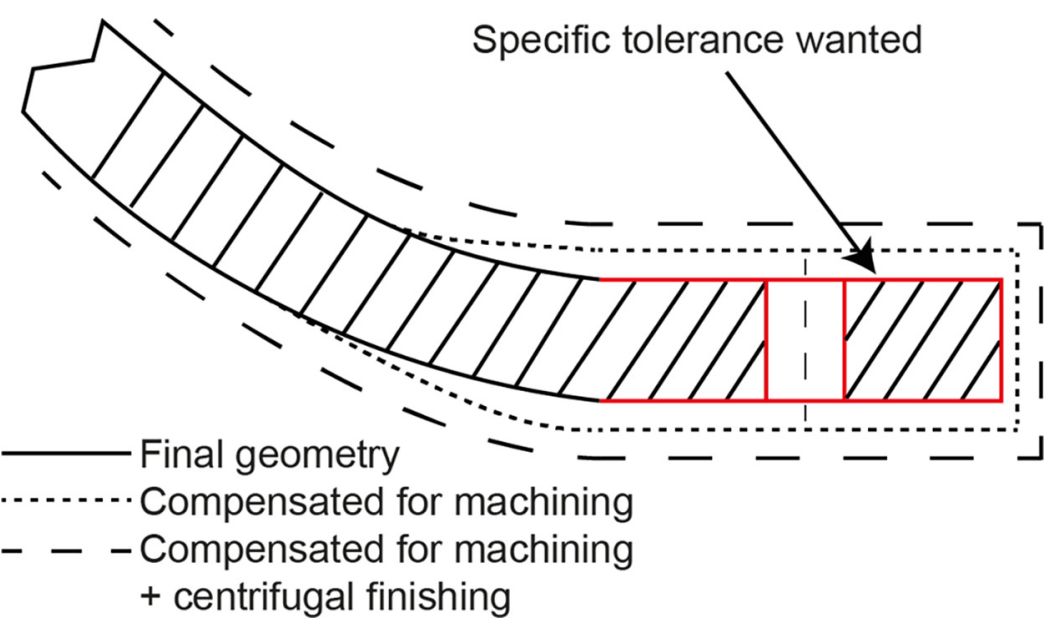


Table 1 Example of the structure of the design rule database for AM machines

\begin{tabular}{|c|c|c|c|c|c|c|}
\hline & \multirow[b]{2}{*}{ Rule } & \multirow[b]{2}{*}{ Description } & \multirow[b]{2}{*}{ Output description } & \multicolumn{3}{|c|}{ Available Machines } \\
\hline & & & & EOS M400 & Arcam Q20 plus & Centrifugal finishing \\
\hline 1 & $\begin{array}{l}\text { Fit within } \\
\text { machine }\end{array}$ & $\begin{array}{l}\text { The part(s) need to fit } \\
\text { within the build area } \\
\text { of the machine }\end{array}$ & $\begin{array}{l}x, y, z(\mathrm{~mm}) \text { : } \\
\text { Size of build chamber. If } x, y, z>0 \text { a rectangular area } \\
\text { is assumed (width, depth, height). If } x, y>0 \& z=0 \text {, } \\
\text { a cylindrical chamber is assumed where } x \text { is radius } \\
\text { and } y \text { height of chamber }\end{array}$ & $400,400,400$ & $350,380,0$ & $250,250,325$ \\
\hline 2 & $\begin{array}{l}\text { Smallest hole } \\
\text { thickness }\end{array}$ & $\begin{array}{l}\text { The smallest inner } \\
\text { distance between two } \\
\text { surfaces }\end{array}$ & $\begin{array}{l}x(\mathrm{~mm}): \\
\text { Smallest hole size allowed }\end{array}$ & 0.2 & 0.4 & 2 \\
\hline 3 & $\begin{array}{l}\text { Smallest wall } \\
\text { thickness }\end{array}$ & $\begin{array}{l}\text { The smallest outer } \\
\text { distance of a wall or } \\
\text { similar }\end{array}$ & $\begin{array}{l}x(\mathrm{~mm}): \\
\text { Smallest wall thickness allowed }\end{array}$ & 0.2 & 0.4 & \\
\hline 4 & $\begin{array}{l}\text { Support } \\
\text { structure for } \\
\text { overhangs }\end{array}$ & $\begin{array}{l}\text { Support structure } \\
\text { necessary to support } \\
\text { overhang features }\end{array}$ & $\begin{array}{l}x \text { (deg), } y \text { (deg): } \\
x=\text { overhang angle allowed for rough surfaces } \\
y=\text { overhang angle allowed for fine surfaces }\end{array}$ & 30,45 & 30,45 & \\
\hline
\end{tabular}

Table 2 Database with description of the material properties that are available

\begin{tabular}{|c|c|c|c|c|c|}
\hline Material & $\begin{array}{l}\text { Machine: } \\
\text { Properties }\end{array}$ & $\begin{array}{l}\text { EOS M400 } \\
\text { Ti64 }\end{array}$ & $\begin{array}{l}\text { EOS M400 + centrifugal } \\
\text { finishing Ti64 }\end{array}$ & $\begin{array}{c}\text { Arcam Q20 plus } \\
\text { Ti64 }\end{array}$ & $\begin{array}{c}\text { Arcam Q20 plus }+ \\
\text { centrifugal finishing Ti64 }\end{array}$ \\
\hline \multirow[t]{6}{*}{ Ti6Al4V } & Static & & & & \\
\hline & - Young's modulus (GPa) & 110 & 110 & 120 & 120 \\
\hline & - Poisson's ratio (-) & 0.3 & 0.3 & 0.3 & 0.3 \\
\hline & - Density $\left(\mathrm{kg} / \mathrm{m}^{3}\right)$ & 4,410 & 4,410 & 4,430 & 4,430 \\
\hline & - Yield Strength (MPa) & 800 & 800 & 1,020 & 1,020 \\
\hline & - Ultimate Strength (MPa) & 860 & 860 & 951 & 951 \\
\hline Ti6Al4V & $\begin{array}{l}\text { Fatigue } \\
\text { - Stress at failure (MPa) as } \\
\text { function of number of cycles }\end{array}$ & $2797.5^{*} x^{-0.161}$ & $1286.7^{*} x^{-0.051}$ & $1683.6^{*} x^{-0.156}$ & $1463.1 * x^{-0.107}$ \\
\hline
\end{tabular}

number is better and an $\mathrm{MF} \leq 0$ means that the rule is fully satisfied. The rules are categorised into compulsory and preferred, where the MF of the compulsory must be below zero while the preferred are instead used as input to the cost model.

The design rules are evaluated based on measures on an STL representation of the manufacturing geometry exported by the MM. An example of a compulsory rule is that the component must fit into the build chamber (Rule 1 in Table 1). The MF is then calculated as how far outside the chamber the component is, with a negative number means that the part fits within. In a similar manor, measured hole (Rule 2) and wall thicknesses (Rule 3) are compared to the minimum requirements defined in the design rules database. Overhang constraint (Rule 4) is defined as a preferred rule and instead of a direct constraint the amount of support structure needed influences the cost of the component. The actual support structure for manufacturing is not generated, instead where support structure is necessary is predicted by an analyse of overhang angles for the current build direction. The amount of support structure is estimated by the prediction where support is needed, the distance to the build plate and a support density factor. New design rules can be added to the database by quantifying the rule into something geometrically measurable and identifying which machine (or other) settings influence the rule.
The cost model used was developed by the manufacturing technology centre (MTC) (The Manufacturing Technology Centre, 2020). The model calculates the cost of manufacturing as a function of AM machine, material, component volume, height and width of the component and amount of support structure necessary. In the model, the AM cost is rounded to the nearest hundred pounds sterling. If post-processing is chosen, a fixed cost (based on a quotation from the subcontractor) is added to the manufacturing cost.

\subsection{Case analysis}

Within the implemented framework, the final performance of the component is analysed in the form of an FE-analysis, used to evaluate the structural performance of the component (as shown in Figure 3). The user must set up the case-specific load cases using a commercial software in an initial phase, thereafter the MM automatically handles the geometrical updates (Figure 4). Based on the selection of material, AM machine and post-process options, the material properties are automatically received from the AM database and used in the calculations.

The results of the structural evaluation are separated into static and fatigue results, where the maximum von Mises stress for the static load cases is compared to the yield strength, and 
for the fatigue cases, it is compared to the fatigue limit for a defined number of load cycles.

\section{Case study}

The proposed method is evaluated by implementing it in a case study in which a structural aircraft component is designed and optimized. The feasibility is measured by how well the method can design the component with the aim of minimising the mass and the manufacturing cost. In addition to the quantitative measurements of the single design case, a qualitative discussion of the properties of the method is presented in Section 5.

\subsection{Studied component}

The component in the case study is a part of the aircraft cargo door structure placed between an actuator used for opening and closing the cargo door and the door structure itself, see

Figure 6 Overview of the aircraft cargo door and the studied component

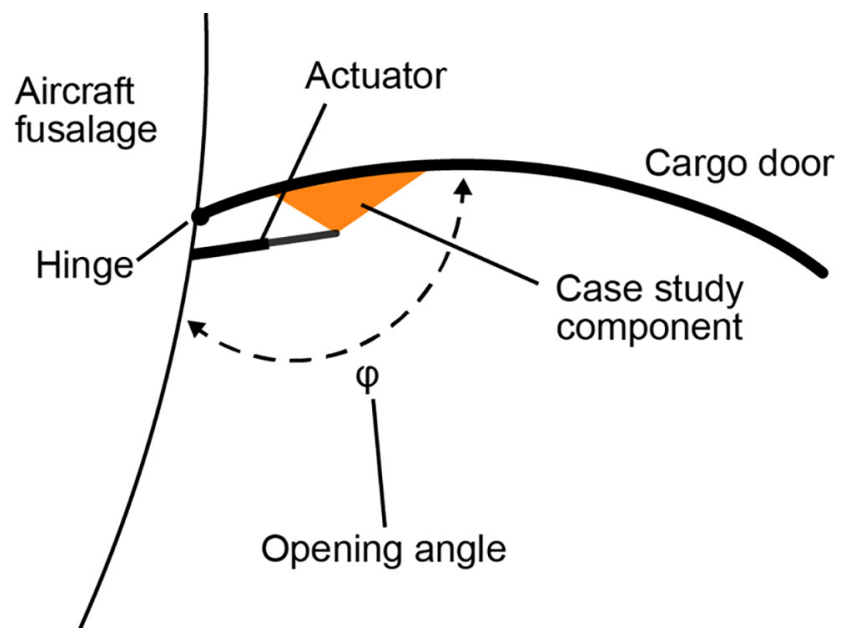

Figure 6. Rivets are used as connectors between the cargo door and the component and a bolt and bearings connect the component to the actuator. In three areas, the positions of the rivets are given, while in two other areas rivets can be placed freely. The positions of the bolt and bearings are given as design inputs (Figure 7).

The component is designed to be manufactured in Ti-6Al$4 \mathrm{~V}$, with the goal of minimising mass and cost while respecting a variety of load cases, including fatigue. Two different AM machines (Arcam Q20 plus and EOS M400, hereafter referred to as Arcam and EOS) could be used for manufacturing, and therefore they were evaluated and compared. Post-processing of the component in term of centrifugal finishing can be used to improve the fatigue properties, and also to reduce the mass. This option is also explored.

Before implementation of the framework presented in Section 3, TO was used to create an initial concept. As presented in Section 3.1, functional decomposition was used to break down the design into sub solutions. In this case the functions can be describes as attach to other components and transfer load. Two types of attachments, rivet and bolt (connection to actuator) are used in the case study. Transfer of loads is performed via trusses which can variate in shape, size and position. Based on the TO result, the MM was created by manual placement of points where attachments and intersections in the truss structure should be placed. These points are only used as references during instantiation of the component and the actual design points can be varied in relation to the references with parameters included in the templates. Since all the geometrical elements and parameters are included in templates, after reference selection, the process is automatic. The parameters modify the placement, shape and size of the trusses in the structure and the placement of the rivets that attach the component to the cargo door. Some of attachment placements were not allowed to be changed while others were free to move on the connection surface. Since it is not known which of the parameters that influence the

Figure 7 Detailed interface between actuator, case study component, and cargo door

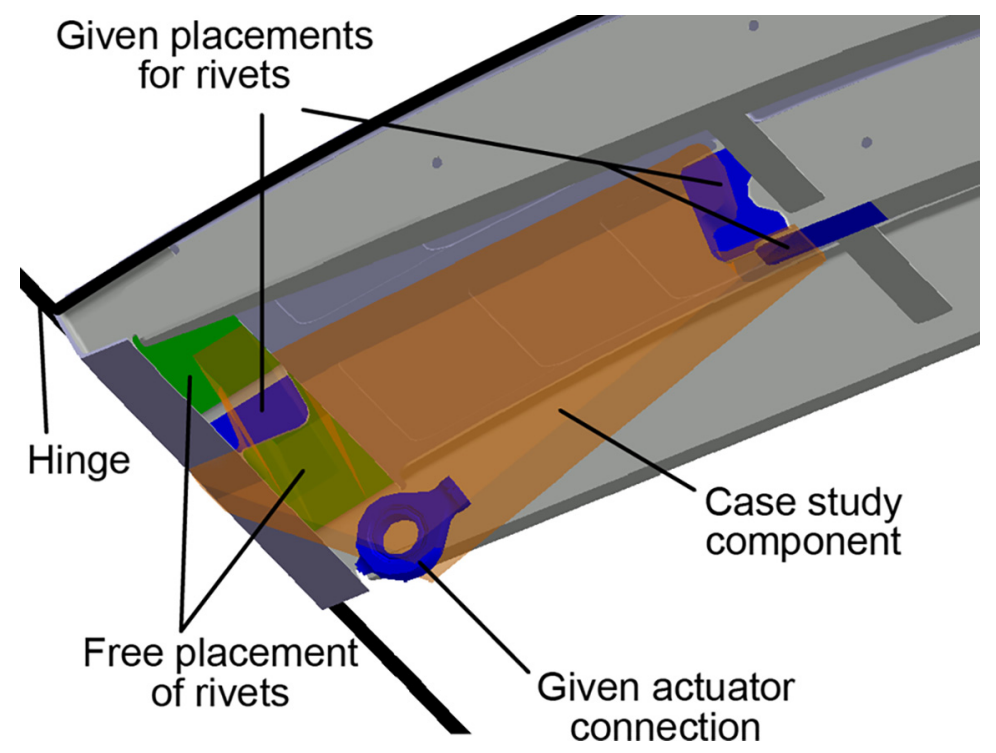


objectives of the design, all parameters included in the templates (except for the position of the attachments not allowed to be changed) could be varied. The MM includes a total of 108 variables for modification of the geometry. A picture of the result from the TO together with an overview of the cross sections in the parametric CAD model, is shown in Figure 8. Figure 9 show a clearer view of the component together with information about the attachments.

\subsection{Multidisciplinary optimisation}

The component is designed by combining the framework presented in section 3 with an MDO framework. The MDO framework is formulated as a distributed framework consisting of two levels. A main level alters the manufacturing setup in the form of AM machine, post-processing option and material. At the second level, the geometry parameters, $\mathbf{x}$, and manufacturing variables, $\mathbf{y}$ (such as build placement and direction), are modified. The MDO framework is described, according to the extended design structure matrix representation (Lambe and Martins, 2012), in Figure 10. In the figure, the rounded dark grey boxes represent multidisciplinary analyses or optimisations, whereas the grey rectangles are computer analyses or processes. White parallelograms are variables or information, and the wide grey lines describe the information flow. Finally, the thin black lines describe the order in which different processes are performed.

The different processes' outputs, such as component mass, manufacturing cost and functional performance, are stored and used as optimisation objectives and constraints. When an MDO of a specific setup is performed, one or several Paretooptimal designs are identified and stored (in the multidisciplinary design analysis block) to allow for comparison between different manufacturing setups.

For a given machine setting, the optimisation in the framework aims to minimise mass and cost while considering the structural and manufacturing constraints. The cost is calculated by the cost model described in Section 3.3 while the mass is extracted from the MM. During the optimisation, geometrical parameters $\mathbf{x}$, presented in Section 4.1, and manufacturing parameters $\mathbf{y}$ are varied. The manufacturing parameters consist of five parameters that control the position and orientation of the component within the build chamber. Design rule 1-3 from Table 1 are used as constraints while design rule 4 (overhang constraint) is included in the cost model as described in Section 3.3. The optimisation formulation is shown in equation (1).

$$
\begin{aligned}
& \operatorname{Min} F(\mathbf{x})=\left\{f_{1}(\mathbf{x}), f_{2}(\mathbf{x}, \mathbf{y})\right\} \quad\left(f_{1}=\text { mass, } f_{2}=\text { cost }\right) \\
& \text { S.tg } \operatorname{tg}_{1}(\mathbf{x})<s_{\max , \text { static }} \quad \text { (static structural constraint) } \\
& g_{2}(\mathbf{x})<s_{\text {max }, \text { fatigue }} \quad \text { (fatigue structural constraint) } \\
& g_{3}(\mathbf{x}, \mathbf{y}) \leq 0 \quad(\text { design rule } 1) \\
& g_{4}(\mathbf{x}, \mathbf{y}) \leq 0 \quad(\text { design rule } 2) \\
& \left.g_{5}(\mathbf{x}, \mathbf{y}) \leq 0 \quad \text { (design rule } 3\right) \\
& x_{i, \min } \leq x_{i} \leq x_{i, \max }(\text { geometry parameters, } i=1,2, \ldots, 108) \\
& \left.y_{i, \min } \leq y_{i} \leq y_{i, \max } \text { (manufacturing settings, } j=1,2, \ldots, 5\right)
\end{aligned}
$$

Problem (1) was solved four times, one for each manufacturing setting (i.e. type of AM machine, and with or without postprocessing). A multi-objective genetic algorithm (MOGA-II) (Poles, 2003) was used to find optimal solutions for the design problem. For each of the four optimisations, a population size of 100 designs was used. The optimisation was run for 80 generations, resulting in a total of 8,000 design evaluations. The use of a genetic algorithm cannot prove optimality; however, an indication that no better solution exists can be seen by studying the improvement in objective values. For all four setups, the lowest cost was found within generations 10-18 while the minimum mass (rounded to nearest 0.1 gram) was found in generations 59-72. Thus, no improvements in neither of the objectives were seen during the final 8 generations (Table 3).

\subsection{Results}

Although the optimisation was formulated as a multiobjective problem (minimise mass and cost) two of the four optimisations (EOS and Arcam with centrifugal finishing)

Figure 8 Cross-sections, splines, and trusses in the MM created from the TO result

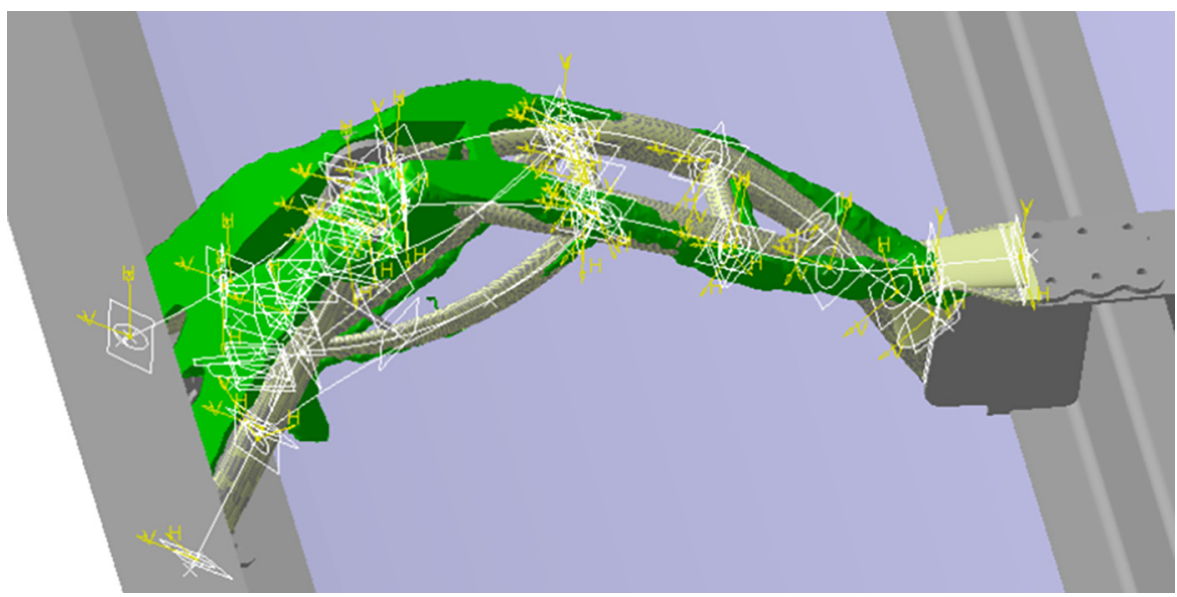


Figure 9 CAD model of the case study component with hidden points, planes and cross-sections

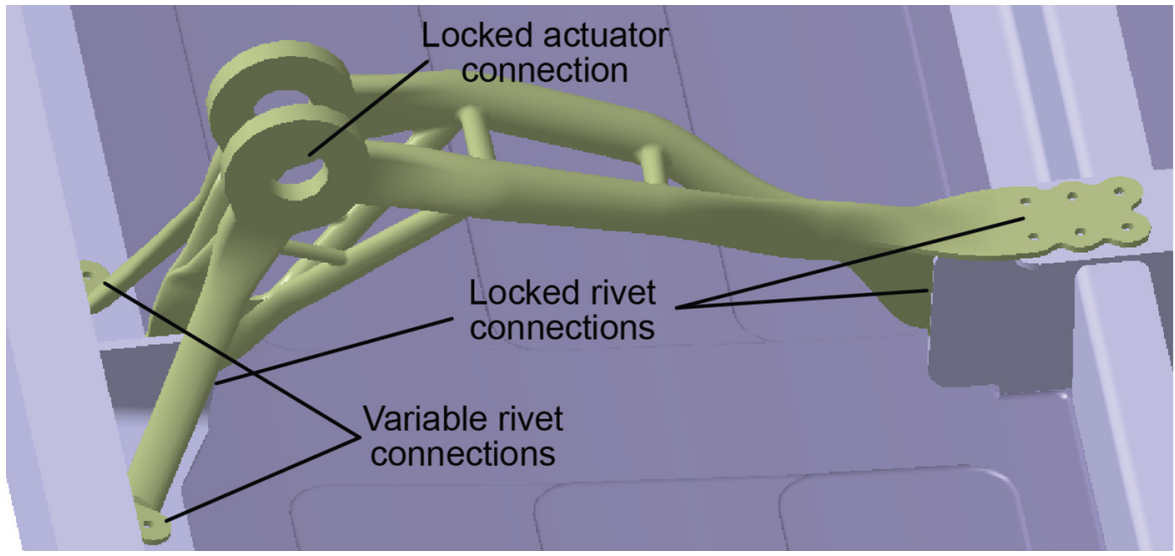

Figure 10 Optimisation framework applied in the case study

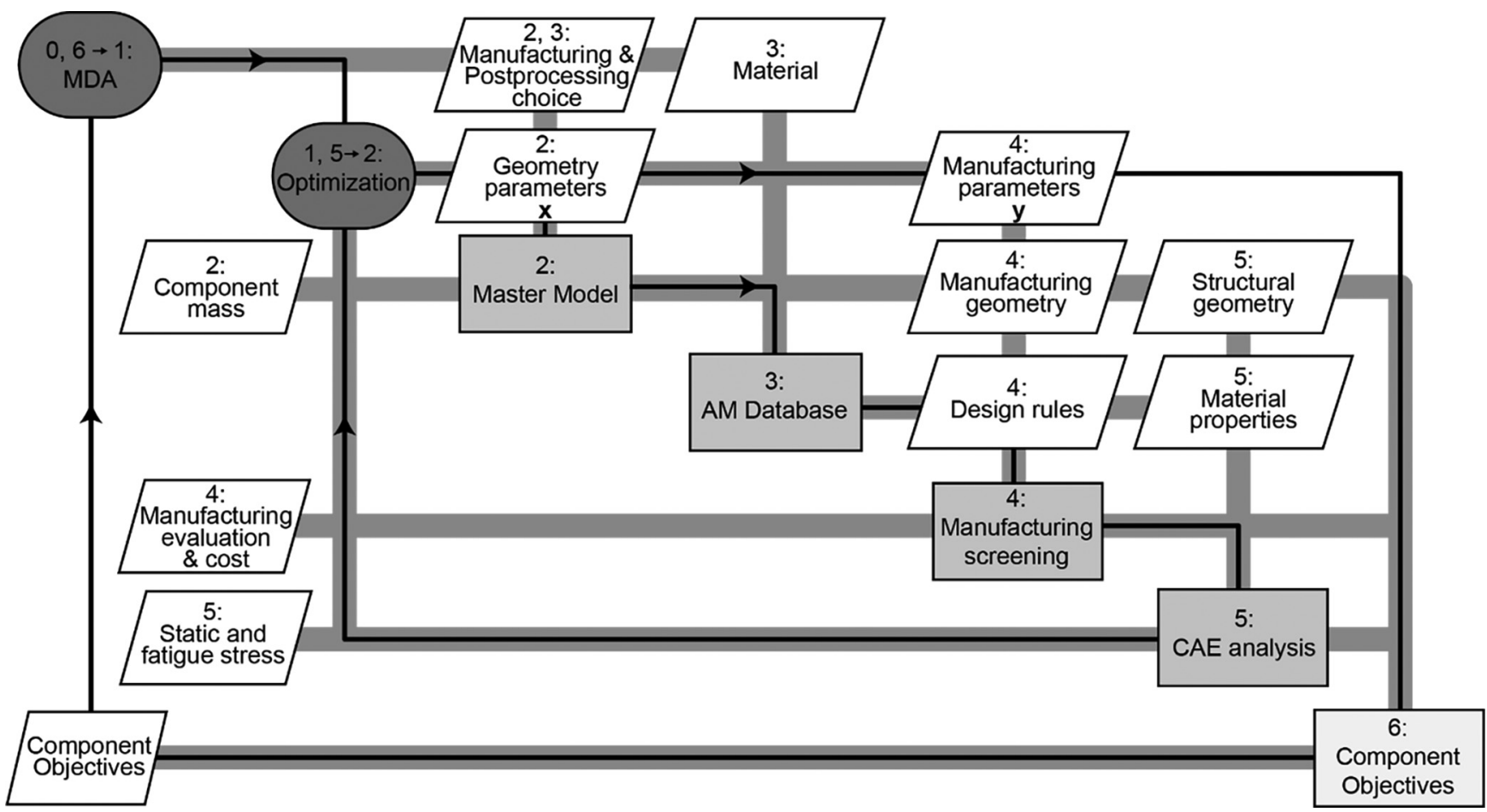

Table 3 Compilation of the generation in which the optimal designs for the two objectives were found

\begin{tabular}{lcc}
\hline Manufacturing setup & $\begin{array}{c}\text { Min mass found } \\
\text { in generation }\end{array}$ & $\begin{array}{c}\text { Min cost found } \\
\text { in generation }\end{array}$ \\
\hline Arcam & 63 & 13 \\
Arcam + centrifugal finishing & 72 & 10 \\
EOS & 59 & 14 \\
EOS + centrifugal finishing & 65 & 18 \\
\hline
\end{tabular}

resulted in one design optimal both in cost and weight. For manufacturing with EOS and Arcam without centrifugal finishing, two Pareto-optimal solutions were found. The objective values for all the six optimal solutions for the four alternative manufacturing setups are shown in Figure 11.

At first glance, all the optimal designs look very similar. This is seen in Figure 12, where the variations between the optimal geometries are shown, as the six obtained optimal design are plotted over each other in three different views. A parameter study of the optimal solutions clearly shows that the fatigue loads are limiting the optimisation and therefore better fatigue properties result in lighter designs. It is also 
Figure 11 Visualisation of the object values for the optimal configurations for the different manufacturing setups

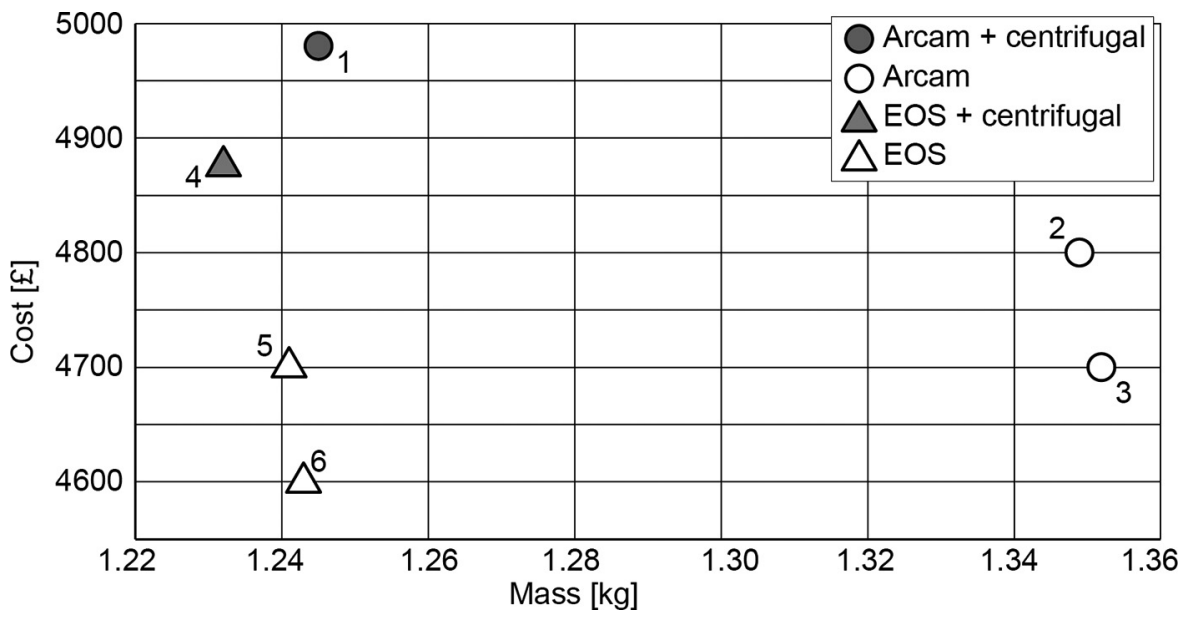

Figure 12 Variations between the optimal solutions seen in three different views. The different grey lines represent the small deviations between the six optimal solutions presented in Figure 11

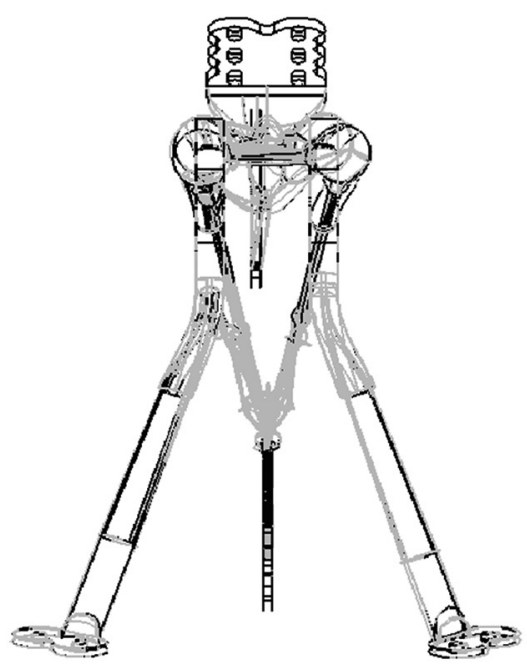

seen that, generally, lower weight also results in lower cost. Another factor that may influence the lack of several Paretooptimal solutions is that the cost model rounds off to the closest hundred pounds sterling.

A significant difference between manufacturing of the component in the Arcam and EOS printers is the build direction. The larger build chamber in the EOS printer allows a build direction in which the component is lying down, while it must stand up in the Arcam machine. This results in both a shorter build time and lower cost in the EOS machine compared to the Arcam machine. The geometrical difference is mainly that better material properties (EOS better than Arcam, and centrifugal finishing better than no centrifugal) results in a slimer design with a lower weight. Figures 13 and 14 show the AM setup with the component shown in red, the build chamber in yellow, and a visual representation of the support structure (not the actual structure) in green.
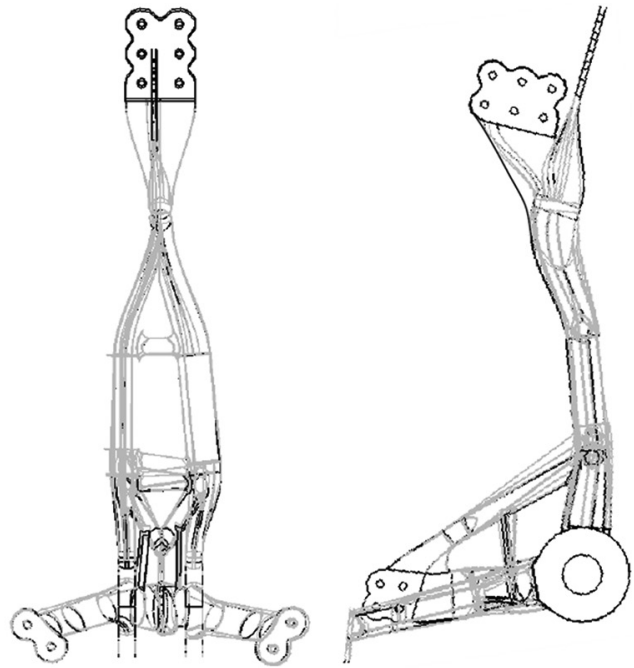

\subsection{Results comparison}

To evaluate the performance of the proposed design method, the results of the case study are compared to a conventionally designed and manufactured component (milled in aluminium) and a design optimised using traditional $\mathrm{TO}$ and $\mathrm{CAD}$ methods. The traditionally designed geometry is based on the same TO result as the one in this study, the final design is made by a design engineer which manually recreated the model in a CAD software. The design is similar to the ones created in this study but not as optimised. With more time and/or a more careful engineer it is possible that this design could have become lighter and thereby probably also cheaper to manufacture. As seen in Table 4, the method implemented in this study was able to reduce the weight significantly compared to the manually designed. While a similar result can be achieved with a manual approach the method presented in this study accomplish it with a simple CAD interaction part which then is automatically improved. Finally, a version of the component 
Figure 13 AM setup for Arcam printer (Design 1)

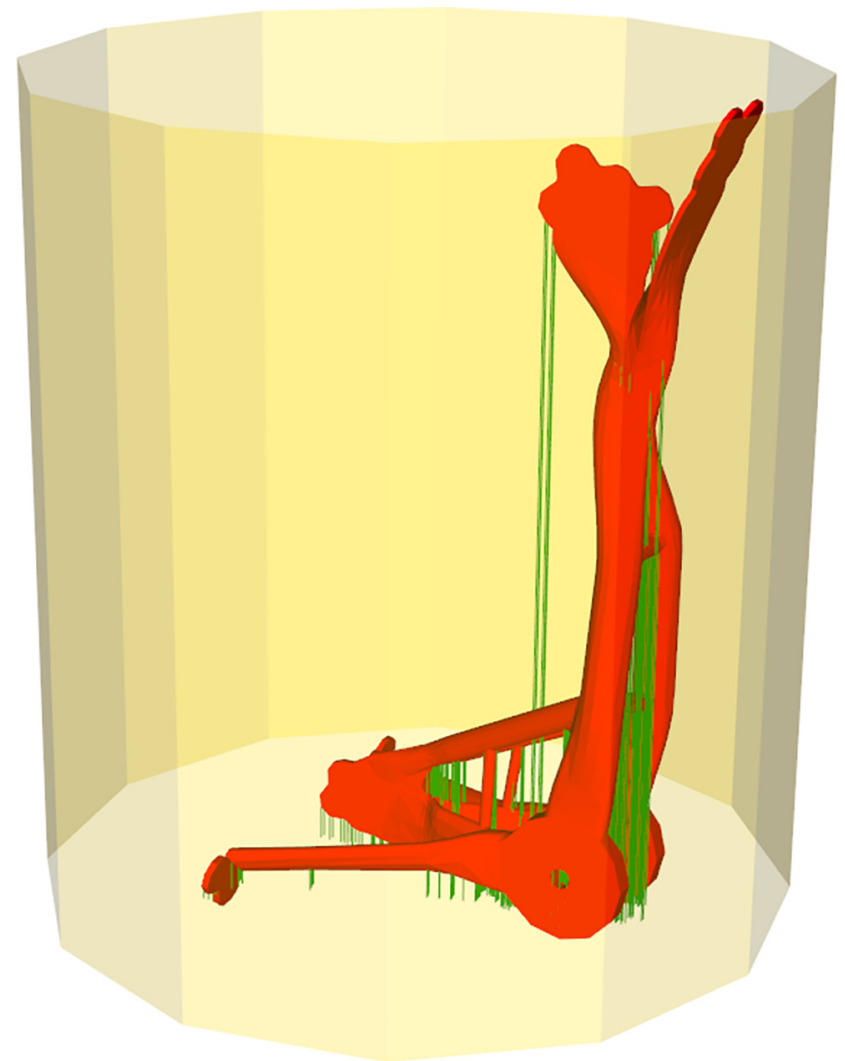

was manufactured and post-processed using centrifugal finishing, as shown in Figure 15.

\section{Discussion}

This paper proposes a design method that combines component design with AM machine selection and manufacturing setup. The method is evaluated and illustrated by applying it to a real industrial problem: designing a structural aircraft component. By combining the proposed method with an optimisation algorithm, the weight and manufacturing cost are minimised while structural loads and manufacturing design rules are considered. Below, Section 5.1 discusses the case study performed; then, based on the case study and future potential, the method is discussed from a generic perspective and how it can be applied in different settings.

\subsection{Case study}

The component in the case study was chosen to be representative of a structural component within the aeronautical industry, which is one of the areas where AM has shown the greatest potential. Since the component is only used to distribute structural loads and has predetermined interfaces with other components, the design freedom is not so large. Hence an approach using TO for concept generation seems natural for this application. Although the creation of the MM is supported by CAD templates, the work to create the $\mathrm{MM}$ is one of the most time-consuming steps in the
Figure 14 AM setup for EOS printer (Design 4)

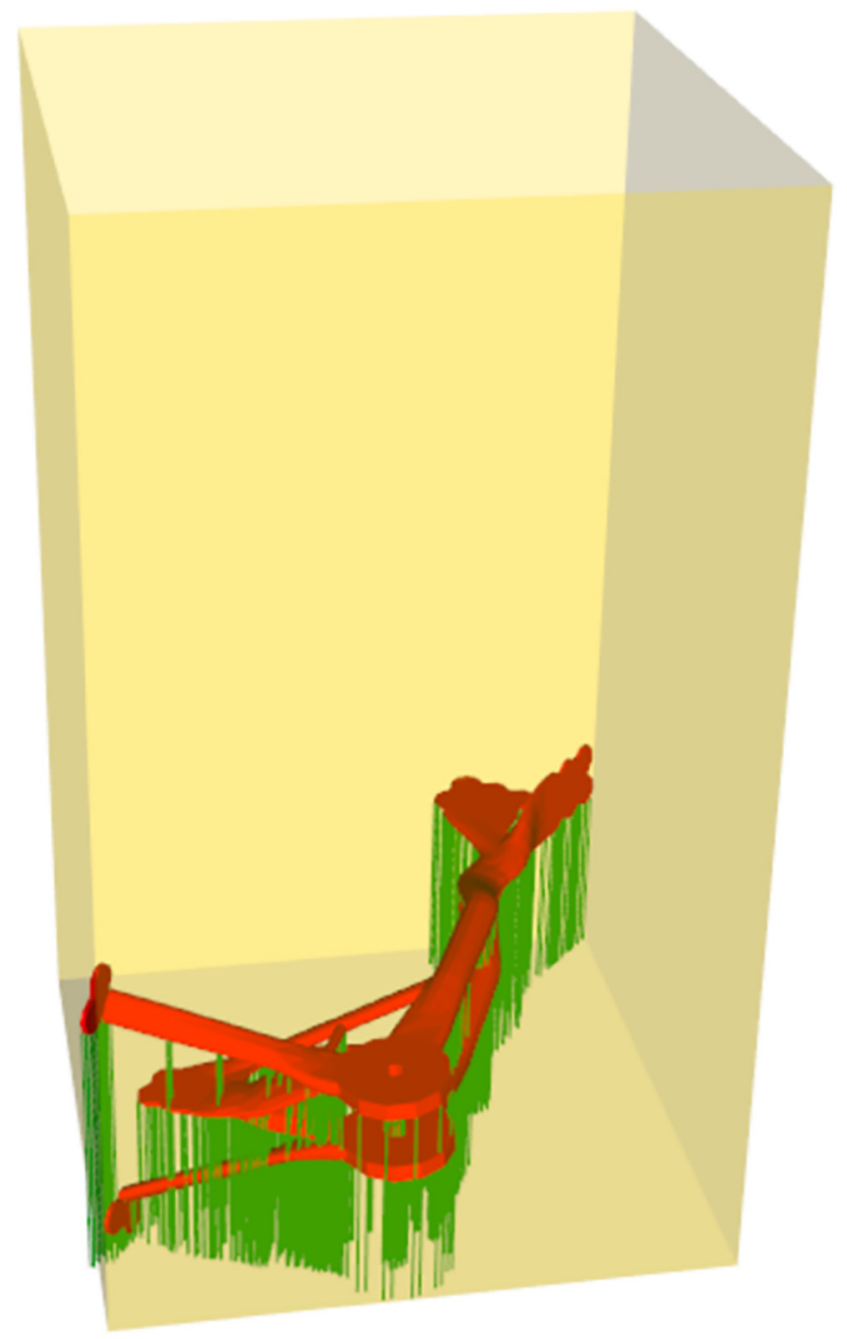

method. A challenge is also which CAD templates that best represent the result achieved from a TO. More alternative templates for recreation of different kind of TO structures can relatively easy be introduced. It would however be more or less impossible to create an exact copy of the TO shape even though a huge number of various templates are introduced. A large number of templates to choose between would also introduce a more difficult step for selecting which templates and references that should be used. Therefore, future work should be directed towards developing and automating this step.

The most time-consuming step in the process was the optimisation which in this case in total occupied 2-3 weeks. This process is automated but can still be a huge time and computational cost. Although the design freedom is not so large, the optimisation performed becomes complex, involving several design variables, multiple objectives and a non-convex solution space. To solve the problem, a genetic algorithm which is powerful and capable of finding good solutions to complex problems was used. The disadvantage is that a large number of design evaluations are required. Together with the large number of design variables (over 100), the design 
Table 4 Comparison between components designed and manufactured using different methods

\begin{tabular}{|c|c|c|c|c|}
\hline & Conventional $^{\mathrm{a}}$ & Traditional design method $^{b}$ & Design 1 (this study) & Design 4 (this study) \\
\hline $\begin{array}{l}\text { Manufacturing method: } \\
\text { Weiaht: }\end{array}$ & Milled in Aluminium & Arcam + centrifugal & Arcam + centrifugal & EOS + centrifugal \\
\hline Manufacturing cost: & n.a. & $£ 5,738$ & $£ 4,980$ & $£ 4,880$ \\
\hline
\end{tabular}

Notes: ${ }^{a}$ Given by the company providing the case study. ${ }^{b}$ Designed in parallel with this paper using a more conventional method combining topology optimisation with manual CAD interpretation and CAE analyse

Figure 15 Component manufactured by Arcam, post-processed by centrifugal finishing and milled to achieve specific tolerances

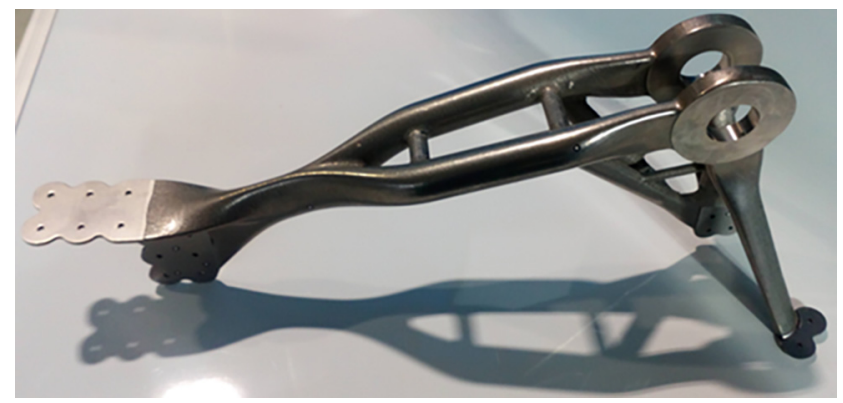

problem becomes difficult and time consuming to solve. For each manufacturing setup, 8,000 design evaluations were used (100 individuals over 80 generations) to solve the problem. The case study shows that no improvement was achieved after generations 59-72, which demonstrates that it is not possible to significantly reduce the number of design evaluations (at least not with this setup).

As seen in Figure 11, few Pareto-optimal solutions were identified in the optimisation. There are two probable reasons for this; the first is that the cost model rounds the predicted cost to the nearest hundred pounds sterling. This reduces the number of almost-similar designs that have a small variation in objective values. The second reason is that the cost model calculates the cost based upon the volume of the component, the height of the component and the amount of support structure. This means the two objectives are strongly correlated since the mass of the component also has a large influence on the cost.

In addition to optimisation of the component for a specific machine, the case study shows that the method enables comparisons between optimised geometries for different manufacturing setups. As one might predict, the addition of centrifugal finishing to the initial manufacturing leads to a lighter but more expensive component, both for EOS and Arcam machines. A comparison between EOS and Arcam manufacturing show that, for this component, EOS is cheaper. This can be traced to the lighter component, because the volume of the manufactured material influences the cost. Another parameter influencing the cost is the height, with a greater height taking a longer time and therefore costing more to manufacture. As seen in Figures 13 and 14, the larger build size in the EOS machine makes it possible to lay the component down, unlike in the Arcam machine, which significantly reduces both the build time and cost.

\subsection{Method}

Compared to existing research, the method presented in this work implements a novel integrated product and process development process. The method integrates knowledge such as design rules, material data, a cost model and manufacturing setup into a holistic automatic framework. Several versions of the geometry created by an MM use manufacturing evaluation of a manufacturing-compensated geometry, in contrast to existing methodologies which first optimise the geometry (with or without design rules) and then compensates the model for manufacturing. Together, this creates great potential to explore different configurations of manufacturing and post-processing without any extra work. In addition to the design and optimisation process, the proposed method can add value by the automation of downstream activities such as AM setup and preparation of manufacturing files.

The case study shows that the proposed method can be applied to a real industrial application and has the potential to automatically compare designs optimised for different AM setups. Based on the case study, two challenges with the method can be pointed out: setup of the geometry and MM, and computational cost for the optimisations. Compared to the time to setup and run the TO, the MM creation takes substantially more time; however, smart use of CAD templates can drastically shorten this time compared to starting modelling from scratch. The time for creation of the MM in the casestudy can be compared to what it would normally take to create a solid, non-smart model. By developing a better, more userfriendly framework for creating the $M M$, the time needed to create it can be reduced, and it will also be easier for new operators to use it.

Five areas for potential improvements to the framework have been identified. Firstly, by developing more and better geometrical templates, larger variations in the geometry can be achieved and it would be easier to create complex intersections between trusses and other geometrical features. A second improvement is the development of more automatic support in the creation of references for the templates. This could be, for example, automatic recognition of intersection features in a TO geometry. To make it more user-friendly for non-related (and the developer) to use, a better user interface would be beneficial. The fourth idea is to use active designrule compensation, whereby CAD templates could automatically adapt to design rules (e.g. orientate a crosssection according to build direction). The computational cost of the system could also benefit from features that automatically orientate according to build direction. This would probably reduce the complexity of the optimisation by weakening the coupling effect between build direction and 
geometry parameters on design rules. A fifth potential for future work is the incorporation of more refined manufacturing evaluation. An advantage of the geometrical rule-based method used in this work is the computational efficiency. The disadvantage is the accuracy which compared to finite element-based simulation of the AM process is not as precise. For future work it would be interesting to combine the rule approach with an AM simulation. To direct exchange the rules with a simulation is probably not computational efficient, instead it is proposed to use a nested framework where the current framework is used to create good solutions and then a simulation can be used to confirm the manufacturability of good solutions.

As seen in the case study, the variation in geometry between the designs that are optimal for different configurations are visually not so large (Figure 12). However, when studying the build orientation, geometry variable values, and the objective values, the difference becomes clear. Limiting parameters in this case are the given interfaces and the purely structural constraint. Application to other domains with more functions and a less well-defined setup would probably lead to larger variations in the obtained geometry. A larger variation in the CAD templates would also lead to larger variations and probably even better objective values. However, all this would probably also create an even more complex optimisation.

As the explicit knowledge in terms of manufacturing design rules and material properties is saved in external databases, it is easy to add new manufacturing methods or combinations of material and post processing methods. Although the uniqueness of this work is the evaluation of manufactureadapted geometries, and the possibility to optimise also the manufacturing setup, it is possible to use the manufacturing evaluation and comparison separately. This functionality could be beneficial to rapidly compare or evaluate which machine should be used to manufacture an already designed component.

\section{Conclusions}

This paper presents a design for an AM method that incorporates the simultaneous design of a component and its manufacturing process, including AM-manufacturing and post-processing parameters. The method includes a MM which enables parametric geometry variations and automatic export of geometries adapted for various simulation processes, such as FEM and manufacturing simulation. Furthermore, a database containing manufacturing design rules and material data for different combinations of AM machine and postprocessing setups is developed. Manufacturability is ensured through the evaluation of geometrical design rules, and a cost model calculates the manufacturing cost. Finally, CAE evaluation is used to ensure that the component fulfils the functional requirements.

The proposed method is showcased and evaluated in a case study in which a structural aircraft component is designed and optimised for manufacture by laser or electron-beam Powder Bed Fusion. Furthermore, the possibility of including a postprocessing step and how this will influence the design and manufacturing cost is also evaluated. Designs optimised for the different manufacturing configurations are automatically achieved and hence different AM machines and postprocessing alternatives could be compared in an efficient manner. The optimisation shows that the build direction, weight of the component and cost of manufacturing varies between the designs that are optimal for different manufacturing configurations. By comparing the results of the case study with the original design (manufactured by conventional methods) and a design accomplished using only topology optimisation and CAD interpretation, it is shown that the method can come up with solutions that are better regarding both performance and cost.

The unique contribution of the presented method is the capability to obtain, without any extra manual work, designs optimised for different AM and post-processing setups. With this as a base, forthcoming studies can be used to further develop computational support to facilitate the manual parts of the CAD process, to improve the evaluation of the manufacturability in more precise ways and to expand the database to include more AM machines, post-process alternatives and materials. To prove the generalisability of the method and computer support, it needs to be applied within different domains and with diverse users.

\section{References}

Adam, G.A.O. and Zimmer, D. (2015), “On design for additive manufacturing: evaluating geometrical limitations", Rapid Prototyping fournal, Vol. 21 No. 6, pp. 662-670.

Amadori, K., Tarkian, M., Ölvander, J. and Krus, P. (2012), "Flexible and robust CAD models for design automation", Advanced Engineering Informatics, Vol. 26 No. 2, pp. 180-195.

Asadollahi-Yazdi, E., Gardan, J. and Lafon, P. (2018), "Toward integrated design of additive manufacturing through a process development model and multi-objective optimization", The International Fournal of Advanced Manufacturing Technology, Vol. 96 No. 9-12, pp. 4145-4164.

Biedermann, M. and Meboldt, M. (2020), "Computational design synthesis of additive manufactured multi-flow nozzles", Additive Manufacturing, Vol. 35

Bikas, H., Stavropoulos, P. and Chryssolouris, G. (2016), “Additive manufacturing methods and modelling approaches: a critical review", The International fournal of Advanced Manufacturing Technology, Vol. 83 Nos 1/4, pp. 389-405.

Blessing, L.T. and Chakrabarti, A. (2009), DRM, a Design Research Methodology, Springer. London,

Blösch-Paidosh, A. and Shea, K. (2019), "Design heuristics for additive manufacturing validated through a user study", Fournal of Mechanical Design, Transactions Of the ASME, Vol. 141 No. 4.

Christensen, P.W. and Klarbring, A. (2009), An Introduction to Structural Optimization, Springer. Linköping,

Das, P. (2016), Optimum Part Build Orientation in Additive Manufacturing for Minimizing Part Errors and Build Time, University of Cincinnati, Cincinnati.

Das, P., Chandran, R., Samant, R. and Anand, S. (2015), "Optimum part build orientation in additive manufacturing for minimizing part errors and support structures", Procedia Manufacturing, Vol. 1, pp. 343-354. 
Gao, W., Ramanujan, D., Ramani, K., Chen, Y., Williams, C. B., Wang, C.C.L., Shin, Y.C., Zhang, S. and Zavattieri, P. D. (2015), "The status, challenges, and future of additive manufacturing in engineering", Computer-Aided Design, Vol. 69, pp. 65-89.

Gibson, I., Rosen, D. and Stucker, B. (2015), Additive Manufacturing Technologies: 3D Printing, Rapid Prototyping, and Direct Digital Manufacturing, second edition, Springer, New York, NY.

Gorguluarslan, R.M.R.M., Gandhi, U.N.U.N., Song, Y.Y. and Choi, S.-K.S.-K. (2017), "An improved lattice structure design optimization framework considering additive manufacturing constraints", Rapid Prototyp. F, Vol. 23 2,

Guessasma, S., Zhang, W., Zhu, J., Belhabib, S. and Nouri, H. (2015), "Challenges of additive manufacturing technologies from an optimisation perspective", International foumal for Simulation and Multidisciplinary Design Optimization, Vol. 6, p. A9.

Jin, Y., Du, J. and He, Y. (2017), "Optimization of process planning for reducing material consumption in additive manufacturing", fournal of Manufacturing Systems, Vol. 44, pp. 65-78.

Kahlin, M., Ansell, H., Basu, D., Kerwin, A., Newton, L., Smith, B. and Moverare, J.J. (2020), "Improved fatigue strength of additively manufactured Ti6Al4V by surface post processing", Int. F. Fatigue, Vol. 134.

Kahlin, M., Ansell, H. and Moverare, J.J. (2017), "Fatigue behaviour of additive manufactured Ti6Al4V, with as-built surfaces, exposed to variable amplitude loading", International Fournal of Fatigue, Vol. 103, pp. 353-362.

Klahn, C., Leutenecker, B. and Meboldt, M. (2014), "Design for additive manufacturing - Supporting the substitution of components in series products", Procedia CIRP, Vol. 21, pp. 138-143.

Klahn, C., Leutenecker, B. and Meboldt, M. (2015), "Design strategies for the process of additive manufacturing", Procedia CIRP, Vol. 36, pp. 230-235.

Kranz, J., Herzog, D. and Emmelmann, C. (2015), "Design guidelines for laser additive manufacturing of lightweight structures in TiAl6V4", fournal of Laser Applications, Vol. 27.

Kumke, M., Watschke, H. and Vietor, T. (2016), "A new methodological framework for design for additive manufacturing", Virtual and Physical Prototyping, Vol. 11 No. 1, p. 3.

Lambe, A.B. and Martins, J.R.R.A. (2012), "Extensions to the design structure matrix for the description of multidisciplinary design, analysis, and optimization processes", Struct. Multidiscip. Optim, Vol. 1

Laverne, F., Segonds, F., Anwer, N. and Coq, M.L. (2014), "DfAM in the design process: a proposal of classification to foster early design stages", Conference 2015 Croat, pp. 1-12,

Lewandowski, J.J. and Seifi, M. (2016), "Metal additive manufacturing: a review of mechanical properties", Annual Review of Materials Research, Vol. 46 No. 1, pp. 151-186.

Lindwall, A. and Törlind, P. (2018), "Evaluating design heuristics for additive manufacturing as an explorative workshop method", Proceedings of the International Destination Conference, Vol. 3 No. 1, pp. 1221-1232.

Liu, J., Gaynor, A.T., Chen, S., Kang, Z., Suresh, K., Takezawa, A., Li, L., Kato, J., Tang, J., Wang, C.C.L., Cheng, L., Liang, X. and To, A.C. (2018), "Current and future trends in topology optimization for additive manufacturing", Structural and Multidisciplinary Optimization, Vol. 57 No. 6, pp. 2457-2483.

Liu, J. and Ma, Y. (2016), "A survey of manufacturing oriented topology optimization methods", Advances in Engineering Software, Vol. 100, pp. 161-175.

Liu, X. and Rosen, D. (2010), “Onotology based knowledge modeling and reuse approach of supporting process planning in layer-based additive manufacturing", International Conference on Manufacturing Automation,

Mani, M., Jee, H. and Witherell, P. (2017), "Design rules for additive manufacturing: a categorization", Proceedings of the ASME Design Engineering Technical Conference,

Perez, B., Hilburn, S., Jensen, D. and Wood, K.L. (2019), "Design principle-based stimuli for improving creativity during ideation", Proceedings of the Institution of Mechanical Engineers, Part C: Fournal of Mechanical Engineering Science, Vol. 233 No. 2, pp. 493-503.

Poles, S. (2003), "MOGA-II: an improved multi-objective genetic algorithm", Technical report 2003-006," Trieste,

Ranjan, R., Samant, R. and Anand, S. (2017), "Integration of design for manufacturing methods with topology optimization in additive manufacturing", f. Manuf. Sci. Eng, Vol. 139 No. 6.

Rosen, D.W. (2014), "Research supporting principles for design for additive manufacturing", Virtual and Physical Prototyping, Vol. 9 No. 4, pp. 225-232.

Rudolph, J.-P. and Emmelmann, C. (2017), "Analysis of design guidelines for automated order acceptance in additive manufacturing", Procedia CIRP, Vol. 60, pp. 187-192.

Schnabel, T., Oettel, M. and Mueller, B. (2017), "Design for additive Manufacturing - Guidelines and case studies for metal applications”, Fraunhofer Inst. Mach. Tools Form. Technol. IWU, Vol. 1, p. 110.

Simpson, T.W. (2017), "Trade-offs with AM", Modern Machine Shop Magazine, pp. 66-68.

Simpson, T.W. and Martins, J.R.R.A. (2011), "Multidisciplinary design optimization for complex engineered systems: report from a national science foundation workshop", F. Mech. Des. Trans. ASME, Vol. 133 No. 10.

The Manufacturing Technology Centre (2020), "Manufacturing technology Centre", available at: www.themtc.org/

Thomas, D. (2009), The Development of Design Rules for Selective Laser Melting, University of Wales, Cardiff.

Vandenbrande, J., Grandine, T. and Hogan, T. (2006), "The search for the perfect body: shape control for multidisciplinary design optimization", 44th AIAA Aerospace Sciences Meeting and Exhibit

Wiberg, A., Persson, J. and Ölvander, J. (2019a), "Design for additive manufacturing using a master model approach", in Proceedings of the ASME 2019 International Design Engineering Technical Conferences and Computers and Information in Engineering Conference IDETC/CIE2019,

Wiberg, A. Persson, J. and Ölvander, J. (2019b), "Design for additive manufacturing - a review of available design methods and software", Manuscr. Submitt. Publ.

Yang, S. and Zhao, Y.F. (2015), "Additive manufacturingenabled design theory and methodology: a critical review", 
The International fournal of Advanced Manufacturing Technology, Vol. 80 Nos 1/4, pp. 327-342.

Zegard, T. and Paulino, G.H. (2016), "Bridging topology optimization and additive manufacturing", Structural and Multidisciplinary Optimization, Vol. 53 No. 1, pp. 175-192.

\section{Further reading}

Asadollahi-Yazdi, E., Gardan, J. and Lafon, P. (2017), "Integrated design for additive manufacturing based on SkinSkeleton approach", Procedia CIRP, Vol. 60, pp. 217-222.
Salonitis, K. and Al Zarban, S. (2015), "Redesign optimization for manufacturing using additive layer techniques", Procedia CIRP, Vol. 36, pp. 193-198.

Wiberg, A., Persson, J. and Ölvander, J. (2018), "“An optimization framework for additive manufacturing given topology optimization results", Tools And Methods for Competitive Engineering - TMCE Proceedings, pp. 533-542.

\section{Corresponding author}

Anton Wiberg can be contacted at: anton.wiberg@liu.se

For instructions on how to order reprints of this article, please visit our website: 